\title{
THE NONHOMOGENEOUS LINEAR DIFFERENCE EQUATION WITH VARIABLE DIFFERENCE INTERVAL ${ }^{1}$
}

TOMLINSON FORT

Certain ordinary linear difference equations whose coefficients can be expressed as factorial series have been treated by Norlund [1]. The corresponding nonhomogeneous equation can then be solved by the difference analogue of the Lagrange variation of parameter method or possibly in other ways. The whole process is very complicated in execution and is at most little more than an existence theorem. The present note treats a general linear difference equation where the constant difference interval is replaced by a variable difference interval. So far as the author knows this is the first time that this has been done. Results include the case where the difference interval is constant as a special case. Also the series used, called general factorial series, include ordinary factorial series as used by Norlund [1] as a special case. It is believed that the author was the first to study such series [2].

1. The equation. We are given a real function of the real variable $x$ which we denote by $h(x)$. The domain of this function is the real continuum. We require that

$$
1 \leqq h(x)<E, \quad \text { a constant. }
$$

We let

$$
\begin{aligned}
h_{0}(x)=0, h_{1}(x) & =h(x), \cdots, h_{j}(x)=h_{j-1}(x)+h_{1}\left(x+h_{j-1}(x)\right) \\
& =\sum_{i=0}^{j-1} h_{1}\left(x+h_{i}(x)\right) .
\end{aligned}
$$

It is immediate that $h_{n+j}(x) \geqq j$ and that $\sum_{j=1}^{\infty} 1 / h_{j}(x)$ diverges. A function that satisfied these conditions is $1+1 / x>a>0$. As a matter of notation with $n, k=0,1,2, \cdots$ we let

$$
\begin{gathered}
\left(x+h_{k}(x)\right)\left(x+h_{k+1}(x)\right) \cdots\left(x+h_{k+m}(x)\right) \\
=\left(x+h_{k+m}(x)\right)^{(m+1)},\left(x+h_{k}(x)\right)^{(0)}=1, \\
h_{k}(x) h_{k+1}(x) \cdots h_{k+m}(x)=h_{k+m}^{(m+1)}(x), h_{k}^{(0)}(x)=1,
\end{gathered}
$$

Received by the editors February 15, 1968 and, in revised form, August 30, 1968.

1 This research was made possible by a grant from National Aeronautics and Space Administration. 


$$
\begin{aligned}
\left(h_{k}(x)-h_{j}(x)\right) & \left(h_{k+1}(x)-h_{j}(x)\right) \cdots\left(h_{k+m}(x)-h_{j}(x)\right) \\
& =\left(h_{k+m}(x)-h_{j}(x)\right)^{(m+1)},\left(h_{k}(x)-h_{j}(x)\right)^{(0)}=1 .
\end{aligned}
$$

We shall study

$$
\begin{aligned}
y(x)=p_{1}(x) y\left(x+h_{1}(x)\right)+p_{2}(x) y & \left(x+h_{2}(x)\right)+\cdots \\
& +p_{n}(x) y\left(x+h_{n}(x)\right)+S(x),
\end{aligned}
$$

where $p_{1}(x), p_{2}(x), \cdots, p_{n}(x)$ and $S(x)$ are each developable into convergent general factorial series, namely series of the type

$$
\sum_{j=0}^{\infty} a_{j}(h(x)) /\left(x+h_{j}(x)\right)^{(j)}, \quad x>a>0,
$$

where $a$ is also a constant. Here $a_{j}(h(x))$ is a polynomial in the $h_{i}$ 's, $i=0,1,2, \cdots$. Certain restrictions which we shall place on $p_{1}(x)$, $p_{2}(x), \cdots, p_{n}(x)$ and on $S(x)$ as well as those placed on $h(x)$ are sufficient, but not proved, necessary for the results of the paper.

2. A heuristic existence theorem. We first assume that

$$
\begin{aligned}
& p_{i}(x)=\sum_{j=1}^{\infty}{ }_{i} d_{j}(h(x)) /\left(x+h_{j}(x)\right)^{(j)} \\
& i=1,2, \cdots, n, \quad x>a>0, \\
& S(x)=\sum_{j=1}^{\infty} C_{j}(h(x)) /\left(x+h_{j}(x)\right)^{(j)}, \\
& C_{j}(h(x)) \not \equiv 0 \text { for all } j \text { 's. }
\end{aligned}
$$

Here ${ }_{i} d_{j}(h(x))$ and $C_{j}(h(x))$ are polynomials in the $h_{i}(x)$ 's, $i=1$, $2, \cdots$ The series are assumed absolutely convergent.

We now let

$$
\begin{aligned}
y(x)=b_{0}(h)+b_{1}(h) /\left(x+h_{1}\right) & +b_{2}(h) /\left(x+h_{2}\right)^{(2)}+\cdots \\
& +b_{j-1}(h) /\left(x+h_{j-1}\right)^{(j-1)}+\cdots .
\end{aligned}
$$

We omit the $x$ in writing $h(x)$. We shall continue to do this.

By means of a simple step-up process we find

$$
\begin{array}{r}
y(x)={ }_{8} B_{0}(h)+{ }_{8} B_{1}(h) /(x+ \\
\left.+h_{s+1}\right)+{ }_{s} B_{2}(h) /\left(x+h_{s+2}\right)^{(2)}+\cdots \\
+{ }_{s} B_{j-1}(h) /\left(x+h_{s+j-1}\right)^{(j-1)}+\cdots,
\end{array}
$$

where

$$
{ }_{8} B_{j-1}(h)=b_{j-1}(h)+{ }_{8} F\left(b_{1}(h), \cdots, b_{j-2}(h), h\right) .
$$


The precise form of $F$ is not important for our present purposes, although given later on.

To see how this step-up process is carried out we illustrate as follows:

We have the identity,

$$
\begin{array}{r}
1 /\left(x+h_{1}\right)=1 /\left(x+h_{s+1}\right)+\left(h_{s+1}-h_{1}\right) /\left(x+h_{s+2}\right)^{(2)}+\cdots \\
+\left(h_{s+r-1}-h_{1}\right)^{(r-1)} /\left(x+h_{s+r}\right)^{(r)}+{ }_{1}^{8} R_{(r+1)},
\end{array}
$$

where

$$
\begin{aligned}
\stackrel{8}{1} R_{(r+1)}= & \left(h_{s+r}-h_{1}\right)^{(r)} /\left(x+h_{1}\right)\left(x+h_{s+r}\right)^{(r)} \\
= & {\left[1 /\left(x+h_{1}\right)\right]\left[1 /\left\{1+\left(x+h_{1}\right) /\left(h_{s+1}-h_{1}\right)\right\} \ldots\right.} \\
& \left.\cdot\left\{1+\left(x+h_{1}\right) /\left(h_{s+r}-h_{1}\right)\right\}\right] .
\end{aligned}
$$

The fact that $\sum_{j=1}^{\infty} 1 / h_{j}$ diverges assures us that ${ }_{1}^{s} R_{(r+1)}$ has the limit zero when $r$ becomes infinite. We remember that $0<a<x$, where $a$ is constant. We also note that ${ }_{1}^{s} R_{(r+1)} \cdot\left(x+h_{s+r}\right)^{(r)} \rightarrow 0$ when $x$ becomes infinite. In other words the infinite series is in this sense (general factorial) asymptotic to $1 /\left(x+h_{1}\right)$. This is not dependent upon the divergence of $\sum_{j=1}^{\infty} 1 / h_{j}$. Also (11) is an identity only dependent upon the nonvanishing of any denominator.

To get a series for $1 /\left(x+h_{2}\right)^{(2)}$ in $h_{s+1}$ we proceed as follows:

We have just proved

$$
1 /\left(x+h_{1}\right)=\sum_{j=1}^{\infty}\left(h_{s+j-1}-h_{1}\right)^{(j-1)} /\left(x+h_{s+j}\right)^{(j)} .
$$

In a precisely similar way we prove

$$
1 /\left(x+h_{2}\right)=\sum_{j=1}^{\infty}\left(h_{g+j-2}-h_{2}\right)^{(j-1)} /\left(x+h_{g+j-1}\right)^{(j)} .
$$

We now multiply each term of (12) into $\left(12^{\prime}\right)$ term by term choosing $g$ as illustrated. Thus $\left(h_{s+t-1}-h_{1}\right)^{(t-1)} /\left(x+h_{s+t}\right)^{(t)}$ is multiplied into $\left(12^{\prime}\right)$ with $g=(s+t+1)$.

We write

$$
\begin{aligned}
1 /\left(x+h_{2}\right)^{(2)}= & 1 /\left(x+h_{s+2}\right)^{(2)}+\left(h_{s+2}-h_{2}\right) /\left(x+h_{s+3}\right)^{(3)} \\
& +\left(h_{s+3}-h_{2}\right)^{(2)} /\left(x+h_{s+4}\right)^{(4)}+\cdots \\
& +\left(h_{s+1}-h_{1}\right) /\left(x+h_{s+3}\right)^{(3)} \\
& +\left(h_{s+3}-h_{2}\right)\left(h_{s+1}-h_{1}\right) /\left(x+h_{s+4}\right)^{(4)}+\cdots \\
& +\left(h_{s+2}-h_{1}\right)^{2} /\left(x+h_{s+4}\right)^{(4)}+\cdots+\cdots
\end{aligned}
$$


We sum by columns and obtain

$$
\begin{aligned}
1 /\left(x+h_{s+2}\right)^{(2)}={ }_{2} A_{0}^{[s]} /\left(x+h_{s+2}\right)^{(2)} & +{ }_{2} A_{1}^{[s]} /\left(x+h_{8+3}\right)^{(3)}+\cdots \\
& +{ }_{2} A_{j-1}^{[s]} /\left(x+h_{8+j+1}\right)^{(j+1)}+\cdots,
\end{aligned}
$$

where

$$
{ }_{2} A_{j}^{[s]}=\sum_{i=0}^{j}\left(h_{s+j+1}-h_{2}\right)^{(j-i)}\left(h_{s+i}-h_{1}\right)^{(i)} .
$$

This series is absolutely convergent as is shown in [2] by consideration of double series. We now make like developments for $1 /\left(x+h_{3}\right)^{(3)}$ etc. We then multiply by the respective coefficients and sum. To show once again, but more generally, how this step-up process is carried out we write

$$
\begin{aligned}
y=b_{0}(h)+ & b_{1}(h)\left[1 /\left(x+h_{8+1}\right)+{ }_{1} A_{1}^{[s]} /\left(x+h_{s+2}\right)^{(2)}\right. \\
& \left.+{ }_{1} A_{2}^{[8]} /\left(x+h_{8+3}\right)^{(3)}+{ }_{1} A_{3}^{[s]} /\left(x+h_{s+4}\right)^{(4)}+\cdots\right] \\
& +b_{2}(h)\left[1 /\left(x+h_{8+2}\right)^{(2)}+{ }_{2} A_{1}^{[s]} /\left(x+h_{s+3}\right)^{(3)}\right. \\
& \left.+{ }_{2} A_{2}^{[s]} /\left(x+h_{8+4}\right)^{(4)}+\cdots\right] \\
+ & b_{3}(h)\left[1 /\left(x+h_{8+3}\right)^{(3)}+{ }_{3} A_{1}{ }^{[s]} /\left(x+h_{s+4}\right)^{(4)}+\cdots\right] \\
+ & b_{4}(h)\left[1 /\left(x+h_{s+4}\right)^{4}+\cdots\right]+\cdots
\end{aligned}
$$

Here we are letting

$$
\begin{array}{r}
{ }_{k} A_{j-1}^{[8]}=\sum_{v=1}^{j}{ }_{k-1} A_{v-1}^{[s]}\left(h_{s+j+k-2}-h_{k}\right)^{(j-v)}, \\
j=1,2, \cdots, k=2,3, \cdots .
\end{array}
$$

We now sum by columns as above getting the $B$ 's of equation (10), where more precisely

$$
{ }_{s} B_{j-1}(h)=\sum_{k=1}^{j-1}{ }_{k} A_{j-k-1}^{[s]} b_{k} \quad \text { and } \quad B_{0}(h)=b_{0}(h) .
$$

We now form $p_{i}(x) y\left(x+h_{i}\right)$. We apply the step-up process to $p_{i}(x)$ and obtain

$$
\begin{gathered}
p_{i}(x)={ }_{i} d_{1} /\left(x+h_{s+t}\right)+\left({ }_{i} d_{2}+{ }_{i}^{t} D_{2}(h)\right) /\left(x+h_{s+t+1}\right)^{(2)} \\
+\left({ }_{i} d_{3}+{ }_{i}^{t} D_{3}(h)\right) /\left(x+h_{s+t+2}\right)^{(3)}+\cdots \\
+\left({ }_{i} d_{j}+{ }_{i}^{t} D_{j}(h)\right) /\left(x+h_{s+t+j-1}\right)^{(j)}+\cdots
\end{gathered}
$$


where

$$
{ }_{i}^{t} D_{j}^{[s]}=\sum_{v=1}^{j-1}{ }_{i} d_{v}(h)_{v}^{t} A_{j-v}^{[8]} .
$$

Here again the series converges absolutely.

Now from (9), noting that $h_{i}(x)+h_{j}\left(x+h_{i}(x)\right)=h_{i+j}(x)$,

$$
\begin{aligned}
y\left(x+h_{i}\right)= & b_{0}\left(h\left(x+h_{i}\right)\right)+b_{1}\left(h\left(x+h_{i}\right)\right) /\left(x+h_{i+1}\right) \\
& +b_{2}\left(h\left(x+h_{i}\right)\right) /\left(x+h_{i+2}\right)^{(2)}+\cdots \\
& +b_{j-1}\left(h\left(x+h_{i}\right) /\left(x+h_{i+j-1}\right)^{(j-1)}+\cdots .\right.
\end{aligned}
$$

We treat this exactly as we treated the series for $y(x)$ and obtain, after replacing $s$ by $n$ which is the order of (5),

$$
\begin{aligned}
y\left(x+h_{i}\right)= & { }_{n} B_{0}\left(h\left(x+h_{i}\right)\right)+{ }_{n} B_{1}\left(h\left(x+h_{i+n+1}\right)\right) \\
& +{ }_{n} B_{2}\left(h\left(x+h_{i}\right)\right) /\left(x+h_{i+n+2}\right)^{(2)}+\cdots \\
& +{ }_{n} B_{j-1}\left(h\left(x+h_{i}\right)\right) /\left(x+h_{i+n+j-1}\right)^{(j-1)}+\cdots .
\end{aligned}
$$

We multiply each term of this expansion into the appropriate development of $p_{i}(x)$ as here illustrated.

$$
\begin{aligned}
& p_{i}(x) y\left(x+h_{i}\right)= \sum_{v=0}^{\infty}\left[{ }_{n} B_{v}\left(h\left(x+h_{i}\right)\right) /\left(x+h_{i+n+v}\right)^{(v)}\right] \\
& \cdot\left[\sum_{j=1}^{\infty}{ }_{i} d_{j}(h) /\left(x+h_{n+j+v}\right)^{(j)}\right] \\
&+\sum_{v=1}^{\infty}\left[{ }_{n} B_{v}\left(h\left(x+h_{i}\right) /\left(x+h_{n+v+i}\right)^{(v)}\right]\right. \\
& \cdot\left[\sum_{j=2}^{\infty}{ }_{i}^{r} D_{j}(h) /\left(x+h_{n+j+v+i}\right)^{(j)}\right], \\
& r=i+n+1+v .
\end{aligned}
$$

We now form $\sum_{i=1}^{n} p_{i}(x) y\left(x+h_{i}\right)$ and obtain a right-hand member for equation (5). The left-hand member, namely $y(x)$, is given by (10) with $s=n$. We now find $b_{0}(h)=0$ and solve successfully for $b_{1}(h)$, $b_{2}(h), \cdots$. We can do this by the uniqueness theorem [2] which tells us that the numerators over the same factorial power are the same. This, of course, is under the assumption that the series converges uniformly, $x>a$, and has the asymptotic character described. We notice that $b_{j-1}$ occurs in the left-hand member over a denominator of degree $(j-1)$ but in the right-hand member of degree at least $j$. This permits successive determination of $b_{j}(h)$ as desired. 
3. A majorant equation. We wish to set up an equation of type (5) of which we know a solution which can be written as a convergent series of type (10) with the coefficient of each term positive and where each of the functions, $P_{1}, P_{2}, \cdots, P_{n}$ and $S$ can also be written as convergent series of type (10) with all coefficients positive. Such an equation is the following:

$$
\begin{aligned}
y(x)= & P_{1}(x) y\left(x+h_{1}\right)+P_{2}(x) y\left(x+h_{2}\right)+\cdots+P_{n}(x) y\left(x+h_{n}\right) \\
& +\bar{S}(x)=Q(x)+\bar{S}(x),
\end{aligned}
$$

where

$$
\begin{aligned}
P_{1}(x) & =P_{2}(x)=\cdots=P_{n}(x)=1 /(n+1) x \\
& =[1 /(n+1)] \sum_{j=1}^{\infty} h_{j-1}^{(j-1)} /\left(x+h_{j}\right)^{(j)}, \\
\bar{S}(x) & =1 / x-[1 /(n+1) x] \sum_{j=1}^{n} 1 /\left(x+h_{j}\right) .
\end{aligned}
$$

First, this equation has the solution $y=1 / x$. Also

$$
1 / x=\sum_{j=1}^{\infty} h_{n+j-1}^{(j-1)} /\left(x+h_{n+j}\right)^{(j)} .
$$

Also if $i \leqq n$

$$
1 /\left(x+h_{i}\right)=\sum_{j=1}^{\infty}\left(h_{n+j-1}-h_{i}\right)^{(j-1)} /\left(x+h_{n+j}\right)^{(j)} .
$$

We notice that all coefficients are positive beyond the first which may be zero. This assures us that no coefficient in $y(x)$ or $Q(x)$ when developed in powers of $1 /\left(x+h_{n+1}\right)$ is negative.

We wish to show that when we develop $\bar{S}$ into a series similar to the above, all coefficients are zero or positive also. We shall show that when we develop

$$
\begin{aligned}
{\left[1 / x-1 /\left(x+h_{n+1}\right)\right]-[1 /(n+1) x] } \\
\cdot\left[1 /\left(x+h_{1}\right)+1 /\left(x+h_{2}\right)+\cdots+1 /\left(x+h_{n}\right)\right]
\end{aligned}
$$

all coefficients are nonnegative. We perform the required developments and obtain

$$
\begin{aligned}
\bar{S}= & 1 /\left(x+h_{n+1}\right)+\sum_{j=2}^{\infty}\left[1 /\left(x+h_{n+j}\right)^{(j)}\right] \\
& \cdot\left[h_{n+j-1}^{(j-1)}-(1 /(n+1)) \sum_{i=1}^{n} \sum_{s=0}^{j-2}\left(h_{n+j-1}-h_{i}\right)^{(s)} h_{n+j-2-8}^{(j-2-s)}\right] .
\end{aligned}
$$


To proceed, we remark that $h_{n+j-1}>j-1$, that $h_{n+j-1}-h_{i} \leqq h_{n+j-1}-h_{1}$ and that

$$
h_{n+j-2}^{(s)} \cdot h_{n+j-2-s}^{(j-2-s)} \geqq\left(h_{n+j-1}-h_{1}\right)^{(8)} h_{n+j-2-8}^{(j-2-8)} .
$$

Whereupon

$$
\begin{aligned}
h_{n+j-1}^{(j-1)}-[1 /(n & +1)] \sum_{i=1}^{n} \sum_{s=0}^{j-2}\left(h_{n+j-1}-h_{i}\right)^{(s)} h_{n+j-2-s}^{(j-2-8)} \\
& \geqq h_{n+j-1}^{(j-1)}-\sum_{s=0}^{j-2}\left(h_{n+j-1}-h_{1}\right)^{(s)} h_{n+j-2-s}^{(j-2-s)} \\
& \geqq h_{n+j-1}^{(j-1)}-\sum_{s=0}^{j-2} h_{n+j-2}^{(s)} h_{n+j-2-s}^{(j-2-8)} \\
& \geqq(j-1) h_{n+j-2}^{(j-2)}-(j-1) h_{n+j-2}^{(j-2)}=0
\end{aligned}
$$

which establishes the desired contention that all coefficients in the expansion of $\bar{S}$ are nonnegative.

4. Conclusion. We now notice that the processes of $\$ 2$ involve only the operations of addition and multiplication. We also notice that the series obtained in $\$ 2$ formally satisfies the equation. By comparing the coefficients in (5) with those in (17), noting absolute uniform convergence and the asymptotic character of the series, we have the following theorem:

TheOREM. The difference equation (5), where $h$ is as described in $\$ 1$ and where

$$
\begin{gathered}
\left|{ }_{i} d_{j}\right| \leqq[1 /(n+1)] h_{n+j-1}^{(j-1)} \\
\text { for } i=1, \cdots, n, \\
\left|C_{j}\right| \leqq h_{n+j-1}^{(j-1)}-[1 /(n+1)] \sum_{i=1}^{n} \sum_{s=0}^{j-2}\left(h_{n+j-1}-h_{i}\right)^{(s)} h_{n+j-2-s}^{(j-2-s)}, \\
j \geqq 2,\left|C_{1}\right| \leqq 1
\end{gathered}
$$

and $C_{j}$ not zero identically in $x$ for all $j$, has one and only one solution that can be written in the form (10). If $h(z)$ is analytic, this series represents an analytic function over the half plane $x>a$ where the independent complex variable is $z=x+y i$.

The majorant equation, namely (17), can be modified in various ways. The most natural is simply to multiply $\bar{S}$ by a constant getting 
an equation which has $M / X$ as a solution. A second natural way is to change $\bar{S}$ so that $C+M / X$ is a solution.

\section{REFERENCES}

1. N. E. Norlund, Differenzrechnung, Zwölftes Kapital.

2. T. Fort, $A$ series useful in the study of difference equations, J. Math. Anal. Appl 13 (1966), 154-165.

EMORY UNIVERSITY 